\title{
On Lattice Representations with Dcc Posets
}

\section{Gergő Gyenizse $^{1}$}

Received: 9 February 2017 / Accepted: 16 April 2019 / Published online: 20 July 2019

(C) The Author(s) 2019

\begin{abstract}
In this paper, we investigate the class of lattices representable with posets satisfying the DCC condition. We describe a way to decide whether a finite lattice is in this class. We also give a necessary condition for an arbitrary lattice to be in this class. This hints at a notion that would be a weaker version of lower boundedness.
\end{abstract}

Keywords Lattice representation · Lower bounded lattices $\cdot$ DCC posets $\cdot$ D relation

\section{Introduction}

Probably the most basic representation theorem of lattice theory is that every lattice is embeddable into the lattice of equivalences of a large enough set [8]. A complementary result is proved by Schein in [1]: any lattice is representable as a lattice of posets on a set. (The partial orders of a set do not form a lattice with respect to inclusion. The reflexive and transitive binary relations do, however, and when we talk about a lattice of posets, we mean a sublattice of this containing only antisymmetric relations.)

For finite lattices, the equivalance representation can be given on an underlying finite set [5]. This is not true for the poset representation. Sivák in [7] gave a characterization for lattices representable as lattices of posets on a finite set using the notion of small congruences of a lattice (a kind of congruences where every congruence class has at most two elements). In [2], the authors note that this characterization precisely describe the class of finite lower bounded lattices.

This can be seen as a generalization of a theorem of Caspard [3], namely that the lattice of permutations of a finite set is (both lower and upper) bounded. Here the lattice of permutations means the lattice induced by the following order: we fix a linear order on the underlying set, and a permutation $\pi_{1}$ will be smaller than the (distinct) permutation $\pi_{2}$ iff for every pair $i<j$ of the underlying set, $\pi_{1}(i)>\pi_{1}(j)$ implies $\pi_{2}(i)>\pi_{2}(j)$. The result about lattices of posets is a generalization of this because the lattice of permutations can be seen as dually isomorphic to a lattice of posets: for every permutation $\pi$, take the poset $\leq_{\pi}$

Gergő Gyenizse

gergogyenizse@gmail.com

1 Bolyai Institute, University of Szeged, Aradi vértanúk tere 1, 6720 Szeged, Hungary 
defined by $i \leq_{\pi} j$ iff either $i=j$, or $i<j$ and $\pi(i)<\pi(j)$. We leave it to the reader to check that $\pi \rightarrow \leq_{\pi}$ indeed defines an injective dual lattice homomorphism.

Semenova in [6] proves something more general (see Proposition 1.6 and Theorem 4.6): any finite lattice embeddable into the suborder lattice of a poset containing no infinite chain must be lower bounded, and all lower bounded lattices are embeddable into such a lattice. Being embeddable into a suborder lattice of a poset containing no infinite chain means (at least for lattices with a largest element) being representable with posets satisfying both the ascending and descending chain conditions. (A poset satisfies both the DCC and ACC if and only if it has no infinite chain.)

So any lattice can be represented as a lattice of posets, but if we require a finiteness condition for the appearing posets, then we get a much smaller class, which is closely related to the well-known class of lower bounded lattices. This paper is concerned with the following question: what if we require a weaker finiteness condition-only the DCC, but not the ACC-of the posets? Obviously, all lower bounded lattices will be representable in such a way. But it turns out that even among finite lattices, there are representable ones that are not lower bounded.

We give an algorithmic characterization of representable finite lattices, and a general necessary condition. The latter is a weaker version of lower boundedness: instead of $D$ cycles, it forbids cycles of $D$-cycles.

The reader is invited to find a class that is closely related to the class of lattices representable with DCC posets (for example, having the same finite members), thus discovering a connection alike to the one between lower bounded lattices and lattices representable with posets containing no infinite chains.

\section{Preliminaries}

A preorder on a set $X$ is a reflexive and transitive binary relation on $X$. The set of all preorders on $X$ with respect to inclusion forms a lattice, denoted by Pre $X$. Naturally, the meet operation is the set-theoretic intersection, and the join operation is equivalent to

$$
(a, b) \in \alpha \vee \beta \Leftrightarrow \exists k \in \mathbb{N}: \exists c_{1}, \ldots, c_{k} \in X: a=c_{1}, b=c_{k}, \forall i:\left(c_{i}, c_{i+1}\right) \in \alpha \cup \beta .
$$

A sublattice of Pre $X$ that only contains antisymmetric preorders is called a lattice of posets on $X$. A lattice of posets can be considered as a set $\mathfrak{C}$ of posets on the same underlying set such that $\mathfrak{C}$ is closed to set-theoretic intersection and for any $\alpha, \beta \in \mathfrak{C}$ the transitive closure of $\alpha \cup \beta$ is also in $\mathfrak{C}$.

One says that a poset satisfies the descending chain condition (DCC) if there are no elements $a_{1}, a_{2}, \ldots$ such that (with respect to this poset) $a_{1}>a_{2}>\ldots$ The dual of the DCC is the ascending chain condition (ACC). We call a lattice of posets a lattice of DCC-posets if all of its elements satisfy the DCC.

We use the following definitions and statements from [4] to introduce the notions of (and related to) lower bounded lattices.

An element $l$ of a lattice $\mathbf{L}$ is join irreducible if there are no elements $l_{1}, l_{2}<l$ such that $l_{1} \vee l_{2}=l$. It is completely join irreducible if there is a largest element $l_{*}$ among all the elements of the lattice smaller than $l$. If an element is completely join irreducible, then it is also join irreducible, and for finite lattices the converse is also true. The set of the join irreducible elements of $\mathbf{L}$ is denoted by $J(L)$. The element $l$ is join prime if for all $l_{1}, l_{2}$ satisfying $l_{1} \vee l_{2} \geq l$ either $l_{1} \geq l$ or $l_{2} \geq l$. 
The join dependency relation, $D$, is a binary relation on the set of join irreducible elements of $\mathbf{L}$. It is defined by

$$
a D b \Leftrightarrow a \neq b,(\exists c: a \leq b \vee c, \forall d<b: a \not \leq d \vee c) .
$$

If $b$ is completely join irreducible, this is simplified into

$$
a D b \Leftrightarrow a \neq b,\left(\exists c: a \leq b \vee c, a \not \leq b_{*} \vee c\right) .
$$

A lattice $\mathbf{L}$ is lower bounded if there is a finitely generated free lattice $\mathbf{F}_{n}$ and a congruence $\theta \in \operatorname{Con} \mathbf{F}_{n}$ such that all $\theta$-classes have a smallest element, and $\mathbf{L} \approx \mathbf{F}_{n} / \theta$. A lower bounded lattice cannot contain an infinite $D$-path (that is, the graph induced by $D$ does not contain an infinite path).

A $D-$ cycle is a cycle in the graph of $D$ (vertices appearing multiple times is permitted). As a $D$-cycle naturally gives rise to an infinite $D$-path, it also cannot appear in a lower bounded lattice. For finite lattices, the opposite is also true: if the lattice contains no $D$ cycle, it is lower bounded.

Finally, any lower bounded lattice is join semidistributive.

\section{Representation of Finite Lattices}

The problem of whether a finite lattice is representable with DCC posets (i.e. is isomorphic to a lattice of DCC posets) is decidable, as Theorem 1 will show. We will start with the idea for the characterization, then state the theorem and give the (quite technical) proof.

Suppose that we have a finite lattice $\mathbf{L}$, and DCC posets $\delta_{l}$ for each $l \in L$ on the same underlying set, $l \rightarrow \delta_{l}$ is order-preserving, but not a lattice homomorphism. Either it does not commute with meet or it does not commute with join, so there are elements $l_{1}$ and $l_{2}$ such that either $\delta_{l_{1} \wedge l_{2}}<\delta_{l_{1}} \wedge \delta_{l_{2}}$ or $\delta_{l_{1} \vee l_{2}}>\delta_{l_{1}} \vee \delta_{l_{2}}$. In the first case the natural idea is to add edges to $\delta_{l_{1} \wedge l_{2}}$ so that it coincides with $\delta_{l_{1}} \wedge \delta_{l_{2}}$. After this $l \rightarrow \delta_{l}$ may not be order-preserving anymore, hence we need to add any $\delta_{l^{\prime \prime}}$-edge to $\delta_{l^{\prime}}$ for all $l^{\prime}>l^{\prime \prime}$. The second case can be addressed with adding an extra element $x$ to our underlying set for each $\left(y_{1}, y_{2}\right) \in \delta_{l_{1} \vee l_{2}} \backslash\left(\delta_{l_{1}} \vee \delta_{l_{2}}\right)$ edge, and adding either $\left(y_{1}, x\right)$ or $\left(x, y_{2}\right)$ to $\delta_{l_{1}}$, and the other to $\delta_{l_{2}}$.

This process may be infinite, but it has a limit, at which $l \rightarrow \delta_{l}$ is a homomorphism. The problem is that while each step maintains the DCC for the appearing posets, the whole process does not necessarily does so. Note that it is not always a problem if the process if infinite: if we need an extra element $x_{1}$ for the edge $\left(y_{1}, y_{2}\right)$, then an extra element $x_{2}$ for $\left(x_{1}, y_{2}\right)$, then an extra element $x_{3}$ for $\left(x_{2}, y_{2}\right)$, and so on-always a new element for the top segment-then we will get an infinite ascending chain, but no infinite descending one. On the other hand, if we always need a new element for the bottom segment, we will get an infinite descending chain. This shows that it matters that which of $\left(y_{1}, x\right)$ and $\left(x, y_{2}\right)$ goes into $\delta_{l_{1}}$ and which into $\delta_{l_{2}}$.

Unfortunately, the process described above is too complicated, as it needs to correct problems both with meets and with joins. Therefore, we will use a different process: we will concentrate only on the images of the join irreducible elements of $\mathbf{L}$. Instead of the mapping preserving meets and join, we will concentrate on the mapping being order-preserving, and having the following property: if $l \leq l_{1} \vee \cdots \vee l_{k}$ for some $l, l_{1}, \ldots, l_{k} \in J(L)$, then $\delta_{l}$ must be included in $\delta_{l_{1}} \vee \cdots \vee \delta_{l_{k}}$. We will try to ensure this the same way we wanted to 


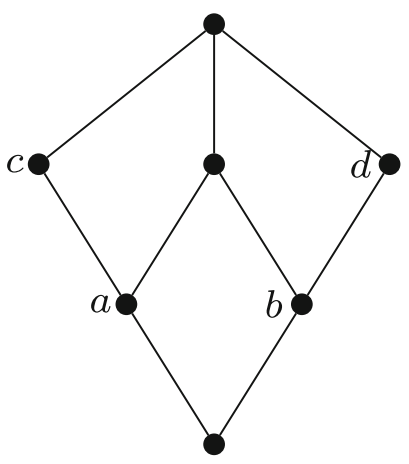

Fig. 1 The lattice $\mathbf{D}_{2}$

ensure that $\delta$ preserved joins, only in this case we need to add $k-1$ extra elements to the underlying set.

To illustrate this process, let us take for example the lattice $\mathbf{D}_{2}$ (Fig. 1). We will only consider the nonzero join irreducible elements of this lattice, which are marked on the figure. Suppose that we have DCC posets $\delta_{a}<\delta_{c}$ and $\delta_{b}<\delta_{d}$. If, for example, $\delta_{c} \leq \delta_{a} \vee \delta_{d}$ does not hold because of an edge $\left(y_{1}, y_{2}\right)$, our process will continously add elements to develop a picture like the one seen on Fig. 2. Note that in Fig. 2, the bottom segments are in $\delta_{a}$ or $\delta_{b}$, while the top segments are in $\delta_{c}$ or $\delta_{d}$. This results in the DCC poset seen on the figure.

Definition 1 For a finite lattice $\mathbf{L}$, let $\mathcal{C}_{\mathbf{L}}$ denote the set of nontrivial join covers of join irreducibles by join irreducibles, i.e. the set

$$
\begin{gathered}
\left\{\left(l, l_{1}, \ldots, l_{k}\right): k \geq 2,\left(l, l_{1}, \ldots, l_{k}\right) \in J(L)^{k+1}: l \leq l_{1} \vee l_{2} \vee \cdots \vee l_{k},\right. \\
\left.l \not \leq\left(l_{1}\right)_{*} \vee l_{2} \vee \cdots \vee l_{k}, l \not \leq l_{1} \vee\left(l_{2}\right)_{*} \vee \cdots \vee l_{k}, \ldots, l \not \leq l_{1} \vee l_{2} \vee \cdots \vee\left(l_{k}\right)_{*}\right\}
\end{gathered}
$$

Theorem 1 A finite lattice $\mathbf{L}$ is isomorphic to a lattice of DCC posets if and only if there is a mapping $s: \mathcal{C}_{\mathbf{L}} \rightarrow J(L)$ satisfying the following:

- for any $\left(l, l_{1}, \ldots, l_{k}\right) \in \mathcal{C}_{\mathbf{L}}, s\left(l, l_{1}, \ldots, l_{k}\right) \in\left\{l_{1}, \ldots, l_{k}\right\}$,

- $s$ is symmetrical in all but the first variable, i.e. for any permutation $\pi \in S_{k}$,

$$
s\left(l, l_{1}, \ldots, l_{k}\right)=s\left(l, l_{\pi(1)}, \ldots, l_{\pi(k)}\right)
$$

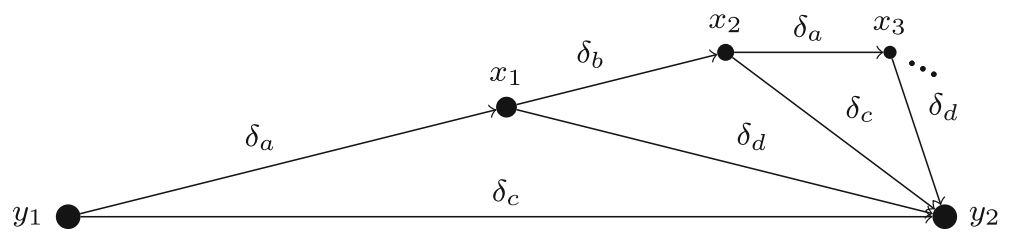

Fig. 2 Edges added by our process beginning with a $\delta_{c}$-edge 
- for the binary relations

$$
T:=\left\{\left(l, l_{i}\right):\left(l, l_{1}, \ldots, l_{k}\right) \in \mathcal{C}_{\mathbf{L}}, s\left(l, l_{1}, \ldots, l_{k}\right) \neq l_{i}\right\}
$$

and

$$
U:=\operatorname{Tr}\left(\{(l, l): l \in L\} \cup\left\{\left(l, l_{i}\right):\left(l, l_{1}, \ldots, l_{k}\right) \in \mathcal{C}_{\mathbf{L}}, s\left(l, l_{1}, \ldots, l_{k}\right)=l_{i}\right\}\right),
$$

the relation $U \circ T$ does not contain a cycle ( $\operatorname{Tr}$ denotes the transitive closure).

Proof $\Leftarrow$ Suppose that there is such a mapping $s$. We will use the process described above, with $s$ telling us that when adding the extra elements to our underlying set, into which poset the top segment will go.

The process will yield a set $X$, partial orders $\gamma_{l} \subseteq X^{2}$ for all $l \in L$ such that the mapping $l \rightarrow \gamma_{l}$ is an injective lattice homomorphism from $\mathbf{L}$ into Pre $X$, and all $\gamma_{l}$ satisfy DCC.

We begin with a set $X^{(0)}=\left\{x_{l, 1}: l \in L\right\} \cup\left\{x_{l, 2}: l \in L\right\}$, and for any $l \in J(L)$ we define the binary relation $\gamma_{l}^{(0)}:=\left\{\left(x_{l, 1}, x_{l, 2}\right)\right\}$ on $X^{(0)}$.

Now we recursively define for all natural $j$ and $l \in J(L)$ the sets $X^{(j)}$ and binary relations $\gamma_{l}^{(j)}$ on $X^{(j)}$. Firstly, $X^{(j)}$ will be a set containing $X^{(j-1)}$, and $\gamma_{l}^{(j)}$ a relation containing $\gamma_{l}^{(j-1)}$. Secondly, for any $\left(l, l_{1}, \ldots, l_{k}\right) \in \mathcal{C}_{\mathbf{L}}$ and any $\left(y_{1}, y_{2}\right) \in \gamma_{l}^{(j-1)} \backslash\left(\gamma_{l_{1}}^{(j-1)} \vee\right.$

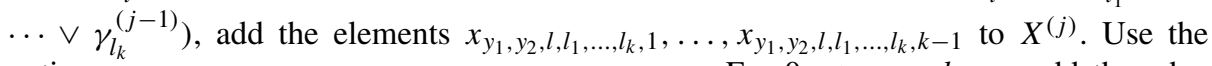

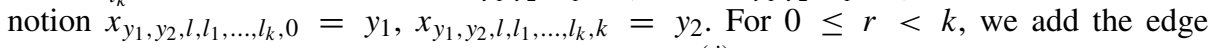
$\left(x_{y_{1}, y_{2}, l, l_{1}, \ldots, l_{k}, r}, x_{y_{1}, y_{2}, l, l_{1}, \ldots, l_{k}, r+1}\right)$ into one of the $\gamma_{l_{i}}^{(j)}$ in a way that exactly one edge goes into each of the $\gamma_{l_{i}}^{(j)}$, and the last edge (that is, $\left.\left(x_{y_{1}, y_{2}, l, l_{1}, \ldots, l_{k}, k-1}, y_{2}\right)\right)$ goes into $\gamma_{s\left(l, l_{1}, \ldots, l_{k}\right)}^{(j)}$.

Finally, set $X=\bigcup X^{(j)}$ and for any $l \in L$ set

$$
\gamma_{l}=\operatorname{Tr}\left(\{(x, x): x \in X\} \cup \bigcup\left\{\gamma_{l^{\prime}}^{(j)}: j \in \mathbb{N}, l^{\prime} \in J(L), l^{\prime} \leq l\right\}\right) .
$$

It is easy to see that all $\gamma_{l}$ are partial orders on $X$, and that $\gamma_{l_{1}}<\gamma_{l_{2}}$ iff $l_{1}<l_{2}$. (The inequality is strict because there is an $l^{\prime} \in J(L)$ such that $l^{\prime} \leq l_{2}$ and $l^{\prime} \not \leq l_{1}$, and then $\left(x_{l^{\prime}, 1}, x_{l^{\prime}, 2}\right)$ is in $\gamma_{l_{2}} \backslash \gamma_{l_{1}}$.)

We will prove that $l \rightarrow \gamma_{l}$ preserves meets and joins. For the "join" part, as $l \rightarrow \gamma_{l}$ is order-preserving, it is enough to show that $\gamma_{l_{1} \vee l_{2}} \leq \gamma_{l_{1}} \vee \gamma_{l_{2}}$ and to do that, it is enough to show that for all $l^{\prime} \in J(L)$ with $l^{\prime} \leq l_{1} \vee l_{2}$, and all $j, \gamma_{l^{\prime}}^{(j)} \subseteq \gamma_{l_{1}} \vee \gamma_{l_{2}}$.

$\mathbf{L}$ is a finite lattice, so each of its elements is a join of join irreducible elements: $l_{1}=$ $p_{1} \vee \cdots \vee p_{m}$, and $l_{2}=q_{1} \vee \cdots \vee q_{n}$. Now as $l^{\prime} \leq l_{1} \vee l_{2}=p_{1} \vee \ldots p_{m} \vee q_{1} \cdots \vee q_{n}$,

$$
\gamma_{l^{\prime}}^{(j)} \subseteq \operatorname{Tr}\left(\gamma_{p_{1}}^{(j+1)} \cup \cdots \cup \gamma_{p_{m}}^{(j+1)} \cup \gamma_{q_{1}}^{(j+1)} \cup \cdots \cup \gamma_{q_{n}}^{(j+1)}\right) \subseteq \gamma_{l_{1}} \vee \gamma_{l_{2}} .
$$

For the "meet" part, take a (non-loop) edge $\left(x_{1}, x_{2}\right) \in \gamma_{l_{1}} \wedge \gamma_{l_{2}}$. If both $x_{1}$ and $x_{2}$ are in $X^{(0)}$, there is an $l^{\prime} \in J(L)$ such that $\left(x_{1}, x_{2}\right)=\left(x_{l^{\prime}, 1}, x_{l^{\prime}, 2}\right)$. Therefore, $l^{\prime} \leq l_{1}$ and $l^{\prime} \leq l_{2}$, so $l^{\prime} \leq l_{1} \wedge l_{2}$, and $\left(x_{1}, x_{2}\right) \in \gamma_{l_{1} \wedge l_{2}}$.

Otherwise, we can assume that both $x_{1}$ and $x_{2}$ are in $X^{(j)}$, but $x_{1}$ is not in $X^{(j-1)}$, so $x_{1}=x_{y_{1}, y_{2}, l^{\prime}, l_{1}^{\prime}, \ldots, l_{k}^{\prime}, r}$, where $y_{1}, y_{2} \in X^{(j-1)}, l^{\prime} \leq l_{1}^{\prime} \vee \cdots \vee l_{k}^{\prime}$, and $\left(y_{1}, y_{2}\right) \in$ $\gamma_{l^{\prime}}^{(j-1)} \backslash\left(\gamma_{l_{1}^{\prime}}^{(j-1)} \vee \cdots \vee \gamma_{l_{k}^{\prime}}^{(j-1)}\right)$. Suppose that $x_{2}=x_{y_{1}, y_{2}, l^{\prime}, l_{1}^{\prime}, \ldots, l_{k}^{\prime}, r^{\prime}}$ for some $r<r^{\prime}$, in which case $\left(x_{1}, x_{2}\right) \in \gamma_{l_{1}} \wedge \gamma_{l_{2}}$ is only possible if

$$
\begin{array}{r}
\left(x_{y_{1}, y_{2}, l^{\prime}, l_{1}^{\prime}, \ldots, l_{k}^{\prime}, r}, x_{\left.y_{1}, y_{2}, l^{\prime}, l_{1}^{\prime}, \ldots, l_{k}^{\prime}, r+1\right)}\right), \ldots,\left(x_{y_{1}, y_{2}, l^{\prime}, l_{1}^{\prime}, \ldots, l_{k}^{\prime}, r^{\prime}-1}, x_{y_{1}, y_{2}, l^{\prime}, l_{1}^{\prime}, \ldots, l_{k}^{\prime}, r^{\prime}}\right) \in \\
\gamma_{l_{1}} \wedge \gamma_{l_{2}},
\end{array}
$$


because any $\gamma_{l}$ on $X^{(j)}$ coincides with the reflexive and transitive closure of $\gamma_{l}^{(j)}$. Hence, $l_{1}, l_{2} \leq l_{r}^{\prime}, \ldots, l_{r^{\prime}-1}^{\prime}$, and thus $l_{1} \wedge l_{2} \leq l_{r}^{\prime}, \ldots, l_{r^{\prime}-1}^{\prime}$, and $\left(x_{1}, x_{2}\right) \in \gamma_{l_{1} \wedge l_{2}}$.

If $x_{2}$ is not of the form $x_{y_{1}, y_{2}, l^{\prime}, l_{1}^{\prime}, \ldots, l_{k}^{\prime}, r^{\prime}}$, then (again because $\gamma_{l}$ on $X^{(j)}$ coincides with the reflexive and transitive closure of $\left.\gamma_{l}^{(j)}\right)\left(x_{1}, x_{2}\right) \in \gamma_{l_{1}} \wedge \gamma_{l_{2}}$ is only possible if $\left(x_{1}, y_{2}\right)$ and $\left(y_{2}, x_{2}\right)$ are both in $\gamma_{l_{1}} \wedge \gamma_{l_{2}}$. As $y_{2}=x_{y_{1}, y_{2}, l^{\prime}, l_{1}^{\prime}, \ldots, l_{k}^{\prime}, k}$, by the previous argument, $\left(x_{1}, y_{2}\right) \in \gamma_{l_{1} \wedge l_{2}}$.

We still need that $\left(y_{2}, x_{2}\right) \in \gamma_{l_{1} \wedge l_{2}}$. If $x_{2}$ is in $X^{(j-1)}$, then we are done by induction. Otherwise, by repeating to $\left(y_{2}, x_{2}\right)$ what we did to $\left(x_{1}, x_{2}\right)$, we will get an $y_{1}^{\prime} \in X^{(j-1)}$ such that it is enough to prove $\left(y_{2}, y_{1}^{\prime}\right) \in \gamma_{l_{1} \wedge l_{2}}$ to prove $\left(y_{2}, x_{2}\right) \in \gamma_{l_{1} \wedge l_{2}}$. That, again, can be done by induction.

We have shown that $l \rightarrow \gamma_{l}$ is strictly order-preserving and is a lattice homomorphism. Therefore, it is a lattice embedding. The only thing left to show is that $\gamma_{1}$ satisfies DCC (1 denoting the largest element of $\mathbf{L}$ ).

For any $l \in L$, the difficulty of $l$ will denote the length of the longest $U \circ T$-path starting from $l$. This is finite because $\mathbf{L}$ is a finite lattice, and $U \circ T$ contains no cycle.

An edge of the type $\left(x_{l, 1}, x_{l, 2}\right)$ will be called an original edge, an edge of the

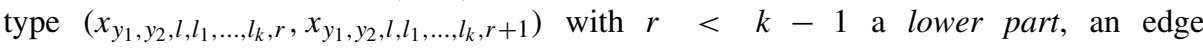

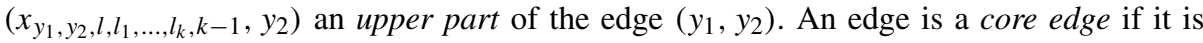
either original, or a lower or upper part of a (core) edge. Thus, each $\gamma_{l}$ is the reflexive and transitive closure of the set of the core $\gamma_{l}$ edges. An edge is an upper edge if it is of the form

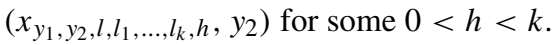

Suppose $\left(a_{i}\right)_{i \in \mathbb{N}}$ is an infinite (strictly) decreasing sequence in $\gamma_{1}$. We can assume that each of the $\left(a_{i+1}, a_{i}\right)$ is a core edge (otherwise, it is the concatenation of core edges,

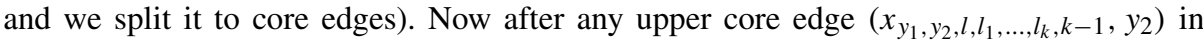
the sequence, the following edge is either another upper core one, or it is the lower core

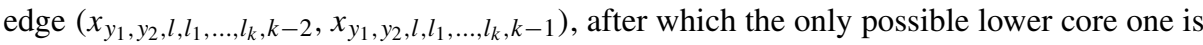

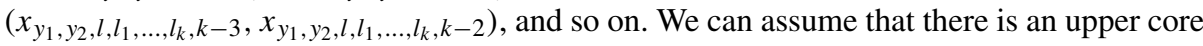

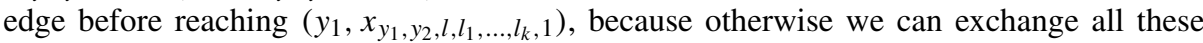
edges to their concatenation $\left(y_{1}, y_{2}\right)$, which is still a core edge. Thus, by concatenating any upper part edge with all the lower part ones following it before the next upper part, we get a sequence of $\gamma_{1}$-edges $\left(b_{i+1}, b_{i}\right)_{i \in \mathbb{N}}$ such that all of those edges are upper edges (though not necessarily cores).

An illustration of this process can be seen on Fig. 3: the edge $\left(a_{2}, a_{1}\right)$ is an upper part (of four parts) of the edge $\left(u, a_{1}\right)$, so $a_{2}=x_{u, a_{1}, l, l_{1}, \ldots, l_{4}, 3}$ for some $\left(l, l_{1}, \ldots, l_{4}\right) \in \mathcal{C}_{\mathbf{L}}$. We choose the smallest $j$ such that there is an element of the sequence $\left(a_{i}\right)_{i \in \mathbb{N}}$ smaller than

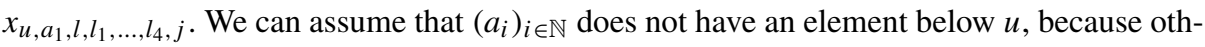
erwise $u$ would equal $a_{h}$ to some $h$, and we could throw out the elements $a_{2}, \ldots, a_{h-1}$ from the sequence. So $j=1$, and we set $b_{2}=x_{u, a_{1}, l, l_{1}, \ldots, l_{4}, j}=a_{6}$. Now, as $\left(a_{7}, a_{6}\right)$ is an upper part of $\left(u, b_{2}\right)$, we choose the smallest $j^{\prime}$ such that there is an element of $\left(a_{i}\right)_{i \in \mathbb{N}}$ smaller than $x_{u, b_{2}, l^{\prime}, l_{1}^{\prime}, \ldots, l_{4}^{\prime}, j^{\prime}}$ (here $l^{\prime}, l_{1}^{\prime}, \ldots, l_{4}^{\prime}$ are such that $a_{7}=x_{u, b_{2}, l^{\prime}, l_{1}^{\prime}, \ldots, l_{4}^{\prime}, 3}$ ). Assuming that no $a_{i}$ is smaller than $v, j^{\prime}$ must be 2 , and we choose $b_{3}$ accordingly.

The following claim presents an immediate contradiction to the existence of such $\left(b_{i}\right)_{i \in \mathbb{N} \text {. }}$

Claim For any $b=x_{y_{1}, y_{2}, l, l_{1}, \ldots, l_{k}, r}$, any path of upper edges ending in $b$ has length not greater than the difficulty of the $l_{j}$ satisfying $\left(x_{y_{1}, y_{2}, l, l_{1}, \ldots, l_{k}, r-1}, b\right) \in \gamma_{l_{j}}$.

Let $d=x_{y_{1}, y_{2}, l, l_{1}, \ldots, l_{k}, r-1}$. 


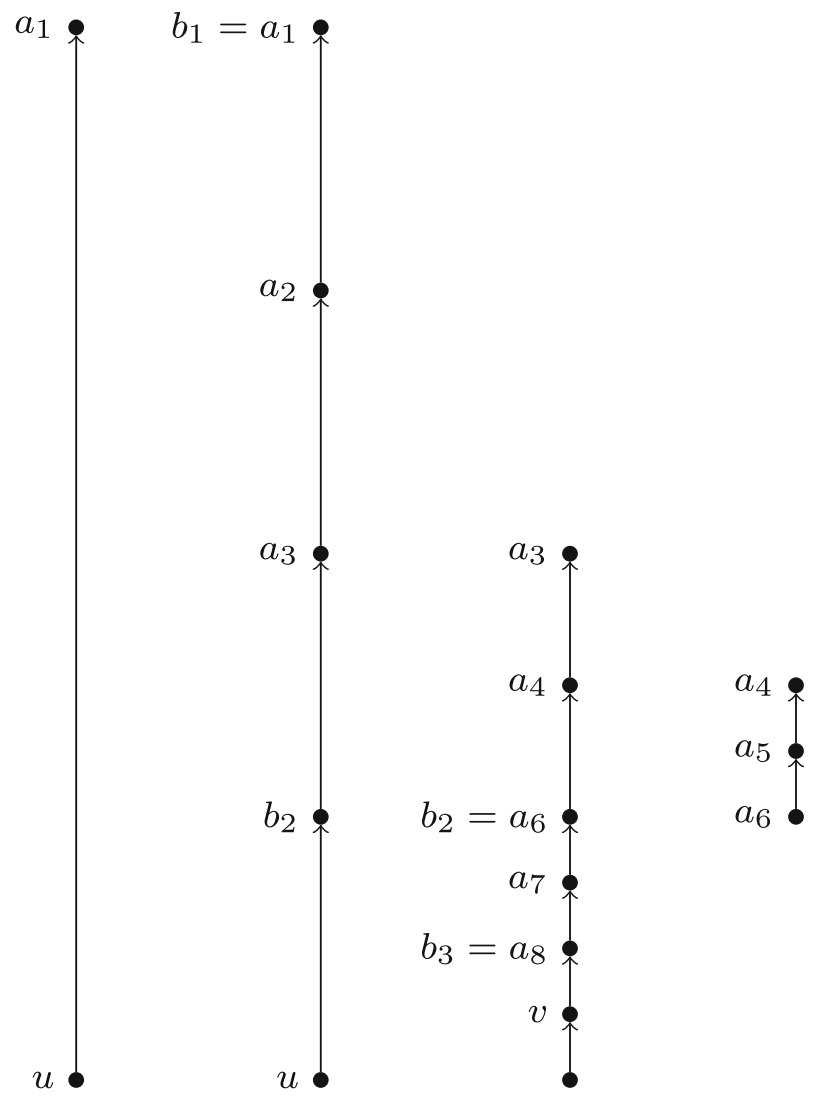

Fig. 3 How to get the sequence $\left(b_{i}\right)_{i \in \mathbb{N}}$ from the sequence $\left(a_{i}\right)_{i \in \mathbb{N}}$. Nodes to the left/right of each other are meant to represent the same element

The claim is proved by induction on the difficulty of $l_{j}$. Suppose first that it is 0 . This means that there is no $T$-edge with source $l_{j}$, consequently, $l_{j}$ is a join prime, and there is

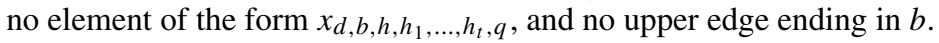

Now suppose that the difficulty of $l_{j}$ is positive. Let $(c, b)$ be an upper edge, thus $c$ is of

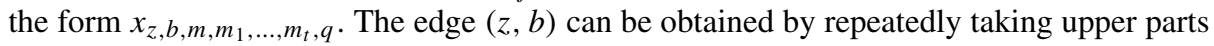

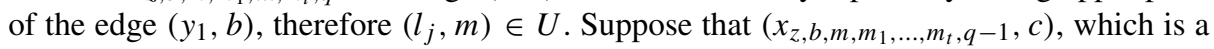
lower part of the edge $(z, b)$, is in $\gamma_{m_{j^{\prime}}}$, then $\left(m, m_{j^{\prime}}\right) \in T$. Thus $\left(l_{j}, m_{j^{\prime}}\right) \in U \circ T$, so the difficulty of $m_{j^{\prime}}$ is smaller than the difficulty of $l_{j}$. By the inductive hypothesis, any path of upper part core edges ending in $c$ has length smaller than the difficulty of $l_{j}$. The claim is proved.

$\Rightarrow$ Assume that $l \rightarrow \gamma_{l}$ is a lattice embedding from $\mathbf{L}$ into Pre $X$. For any edge $\left(z_{1}, z_{2}\right) \in \gamma_{1}$ there is a smallest $k \in L$ such that $\left(z_{1}, z_{2}\right) \in \gamma_{k}$, the weight of this edge.

An edge $(c, d)$ is called contained in the edge $(a, b)$ if both $(a, c)$ and $(d, b)$ are in $\gamma_{1}$, but $(c, d) \neq(a, b)$. It is properly contained if moreover $d<b$.

Let $\left(l, l_{1}, \ldots, l_{k}\right) \in \mathcal{C}_{\mathbf{L}}$, and take an edge $\left(a_{1}, a_{2}\right) \in \gamma_{l} \backslash \gamma_{l_{*}}$, this edge has weight $l$. There are elements $a_{1}=b_{0}, b_{1}, \ldots, b_{r}=a_{2}$ in $X$ such that for all $0 \leq i<r,\left(b_{i}, b_{i+1}\right) \in$ 
$\gamma_{l_{1}} \cup \cdots \cup \gamma_{l_{k}}$. Among these edges there must be at least one with weight $l_{j}$ for all $1 \leq j \leq k$, otherwise

$$
\left(a_{1}, a_{2}\right) \in \gamma_{l} \cap\left(\gamma_{l_{1}} \vee \cdots \vee \gamma_{\left(l_{j}\right)_{*}} \vee \cdots \vee \gamma_{l_{k}}\right) \subseteq \gamma_{l_{*}} .
$$

So each edge with weight $l$ contains at least one edge of weight $l_{1}$, at least one of weight $l_{2}$, etc., and it contains these edges properly with at most one exception.

Now define the mapping $s$ on $\left(l, l_{1}, \ldots, l_{k}\right)$ so that if there are infinitely many edges with weight $l$, but only finitely many contains an edge of weight $l_{j}$ properly, then $s\left(l, l_{1}, \ldots, l_{k}\right)=l_{j}$. If there is no such $j$, then set $s\left(l, l_{1}, \ldots, l_{k}\right)=l_{1}$.

If $\left(l, l^{\prime}\right) \in U \circ T$, then all edges of weight $l$ must contain an edge of weight $l^{\prime}$. Suppose there is a cycle of $U \circ T$ containing the edge $\left(l, l^{\prime}\right)$. There is an $m \in L$ so that $(l, m) \in U$ and $\left(m, l^{\prime}\right) \in T$. All edges of weight $l$ must contain an edge of weight $l$. Starting from an edge $\left(f_{1}, e_{1}\right)$ of weight $l$ we can get the edges $\left(f_{2}, e_{2}\right),\left(f_{3}, e_{3}\right)$ etc., each contained in the previous, and each having weight $l$. Furthermore, for each $j>0$ either $\left(f_{j}, e_{j}\right)$ can be chosen so that $e_{j} \neq e_{j-1}$, or it can only be chosen so that there is an edge of weight $m$ containing $\left(f_{j}, e_{j}\right)$ and contained in $\left(f_{j-1}, e_{j-1}\right)$ that does not properly contain any edge of weight $l^{\prime}$. There are only finitely many such edges of weight $m$. Therefore, the $\left(f_{j}, e_{j}\right)$ edges can be chosen so that the sequence $\left(e_{j}\right)_{j \in \mathbb{N}}$ contains an infinite strictly decreasing subsequence in $\gamma_{1}$, a contradiction.

This theorem gives an algorithm deciding whether $\mathbf{L}$ is representable with DCC posets. The algorithm is in $\mathcal{E} \mathcal{X} \mathcal{P} \mathcal{T} \mathcal{I} \mathcal{M E}$.

Problem 1 Are there real numbers $k$ and $\alpha$ such that for any finite lattice $\mathbf{L},\left|\mathcal{C}_{\mathbf{L}}\right|<k|L|^{\alpha}$ ?

If the answer to this is "yes", then the algorithm is actually in $\mathcal{N P}$. This would not automatically mean that the representability problem itself is in $\mathcal{N P}$ : for that, $\mathcal{C}_{\mathbf{L}}$ needs to also be computable in polynomial time.

Conjecture 1 Deciding whether a finite lattice is representable with DCC posets is an $\mathcal{N} \mathcal{P}$ hard problem.

For comparison, deciding whether a finite lattice is lower bounded is in $\mathcal{P}$, because $D$ is computable in polynomial time.

\section{Representation of Arbitrary Lattices}

For a lattice $\mathbf{L}$, denote with $\mathcal{C} \mathcal{Y}_{\mathbf{L}}$ the set of $D$-cycles of $\mathbf{L}$ consisting of completely join irreducible elements. Introduce a binary relation on $\mathcal{C Y}_{\mathbf{L}}$ :

$$
\begin{aligned}
E_{\mathbf{L}} & :=\left\{\left(\left(\beta_{1}, \ldots, \beta_{l}\right),\left(\alpha_{1}, \ldots, \alpha_{k}\right)\right):\right. \\
\exists i & \left.: \exists j: \alpha_{j+1} \leq \beta_{i} \vee \alpha_{j}, \alpha_{j+1} \not \leq \beta_{i} \vee\left(\alpha_{j}\right)_{*}, \alpha_{j+1} \not \leq\left(\beta_{i}\right)_{*} \vee \alpha_{j}\right\},
\end{aligned}
$$

with the index $j$ meant as modulo $k$ and the index $i$ as modulo $l$.

The following is a necessary condition for a lattice to be representable with DCC posets.

Theorem 2 If $\mathbf{L}$ is representable with DCC posets, then $E_{\mathbf{L}}$ does not contain a cycle. 
Proof Suppose the contrary, that

$$
\underline{\alpha}^{(1)} E_{\mathbf{L}} \underline{\alpha}^{(2)} E_{\mathbf{L}} \ldots E_{\mathbf{L}} \underline{\alpha}^{(t)} E_{\mathbf{L}} \underline{\alpha}^{(1)},
$$

with $\underline{\alpha}^{(i)}=\left(\alpha_{1}^{(i)}, \ldots, \alpha_{k_{i}}^{(i)}\right)$ for all $1 \leq i \leq t$. For all $1 \leq i \leq t$ and $1 \leq j \leq k_{i}$, there is a $\gamma_{j}^{(i)}$ that $\alpha_{j}^{(i)} \leq \gamma_{j}^{(i)} \vee \alpha_{j+1}^{(i)}$ and $\alpha_{j}^{(i)} \not \leq \gamma_{j}^{(i)} \vee\left(\alpha_{j+1}^{(i)}\right)_{*}$, with the index $j$ taken modulo $k_{i}$. By the definition of $E_{\mathbf{L}}$, we may assume that for all $i$ there is an index $m_{i}$ such that $\gamma_{m_{i}}^{(i)}=\alpha_{1}^{(i+1)}$ for all $1 \leq i \leq t$ (taken modulo $t$ ), and furthermore, $\alpha_{m_{i}}^{(i)} \nless \leq\left(\alpha_{1}^{(i+1)}\right)_{*} \vee \alpha_{m_{i}+1}^{(i)}$ is satisfied. Set $\mu$ as the join of all the $\alpha_{j}^{(i)}$.

Start with the inequality

$$
\begin{aligned}
& \alpha_{m_{1}}^{(1)} \leq \gamma_{m_{1}}^{(1)} \vee\left(\alpha_{m_{1}+1}^{(1)} \wedge\left(\gamma_{m_{1}+1}^{(1)} \vee\left(\alpha_{m_{1}+2}^{(1)} \wedge \cdots \vee\left(\alpha_{m_{1}-1}^{(1)} \wedge\left(\gamma_{m_{1}-1}^{(1)} \vee \alpha_{m_{1}}^{(1)}\right)\right)\right)\right)\right)= \\
& \alpha_{1}^{(2)} \vee\left(\alpha_{m_{1}+1}^{(1)} \wedge\left(\gamma_{m_{1}+1}^{(1)} \vee\left(\alpha_{m_{1}+2}^{(1)} \wedge \cdots \vee\left(\alpha_{m_{1}-1}^{(1)} \wedge\left(\gamma_{m_{1}-1}^{(1)} \vee \alpha_{m_{1}}^{(1)}\right)\right)\right)\right)\right)= \\
& \left(\alpha_{1}^{(2)} \wedge\left(\gamma_{1}^{(2)} \vee\left(\alpha_{2}^{(2)} \wedge \cdots \vee\left(\alpha_{m_{2}-1}^{(2)} \wedge\left(\gamma_{m_{2}-1}^{(2)} \vee \alpha_{m_{2}}^{(2)}\right)\right)\right)\right)\right) \vee \\
& \left(\alpha_{m_{1}+1}^{(1)} \wedge\left(\gamma_{m_{1}+1}^{(1)} \vee\left(\alpha_{m_{1}+2}^{(1)} \wedge \cdots \vee\left(\alpha_{m_{1}-1}^{(1)} \wedge\left(\gamma_{m_{1}-1}^{(1)} \vee \alpha_{m_{1}}^{(1)}\right)\right)\right)\right)\right) \text {. }
\end{aligned}
$$

We will call a sequence of $L$-elements $a=c_{0}, \ldots, c_{r}=b$ a realization of the $\alpha_{m_{1}}^{(1)}$ edge $(a, b)$ if the following are satisfied:

- For all $1 \leq s \leq r,\left(c_{s}, c_{s+1}\right)$ is an edge of either $\alpha_{m_{1}}^{(1)}$, or $\alpha_{m_{2}}^{(2)}$, or $\gamma_{j}^{(2)}$ for an $1 \leq j<m_{2}$, or $\gamma_{j}^{(1)}$ for an $1 \leq j<k_{1}, j \neq m_{1}$.

- $\quad$ There are indices $0=r_{0}<r_{1}<\cdots<r_{h}=r$ such that

- For any even $0 \leq h^{\prime}<h,\left(c_{r_{h^{\prime}}}, c_{r_{h^{\prime}+1}}\right) \in \alpha_{1}^{(2)}$, and for any odd $0 \leq h^{\prime}<h$, $\left(c_{r_{h^{\prime}}}, c_{r_{h^{\prime}+1}}\right) \in \alpha_{m_{1}+1}^{(1)}$,

- For even $h^{\prime}$, if $r_{h^{\prime}} \leq s_{1}<s_{2} \leq r_{h^{\prime}+1}$ and $i_{0}$ are such that the set

$$
\left\{s:\left(\left(c_{s}, c_{s+1}\right) \in \alpha_{m_{2}}^{(2)} \vee \exists i: i_{0} \leq i<m_{2},\left(c_{s}, c_{s+1}\right) \in \gamma_{i}^{(2)}\right)\right\}
$$

contains all $s^{\prime}$ satisfying $s_{1} \leq s^{\prime}<s_{2}$ but does not contain $s_{1}-1$ and $s_{2}$, then $\left(c_{s_{1}}, c_{s_{2}}\right) \in \alpha_{i_{0}}^{(2)}$,

- For odd $h^{\prime}$, if $r_{h^{\prime}} \leq s_{1}<s_{2} \leq r_{h^{\prime}+1}$ and $i_{0}$ are such that the set

$$
\begin{array}{r}
\left\{s:\left(\left(c_{s}, c_{s+1}\right) \in \alpha_{m_{1}}^{(1)} \vee\right.\right. \\
\left.\left.\exists i: i \in\left\{i_{0}, i_{0}+1, \ldots, m_{1}-1\right\},\left(c_{s}, c_{s+1}\right) \in \gamma_{i}^{(2)}\right)\right\}
\end{array}
$$

contains all $s^{\prime}$ satisfying $s_{1} \leq j^{\prime}<s_{2}$ but does not contain $s_{1}-1$ and $s_{2}$, then $\left(c_{s_{1}}, c_{s_{2}}\right) \in \alpha_{i_{0}}$.

Note the connection between the definition of a realization and the inequality preceding it: the inequality tells us that each $\alpha_{m_{1}}^{(1)}$ edge will have a realization (an example of a realization can be seen on Fig. 4).

For each $\alpha_{m_{1}}^{(1)}$ edge we choose a single realization by the axiom of choice to get a canonical realization. We obtain likewise canonical realizations for $\alpha_{m_{i}}^{(i)}$ edges for all $1 \leq i \leq t$ (just switch the lower indices everywhere in the definition cyclically by $i-1$ ). If an edge is at the same time an $\alpha_{m_{i_{1}}}^{\left(i_{1}\right)}$ and an $\alpha_{m_{i_{2}}}^{\left(i_{2}\right)}$ edge, it will get a canonical realization both as an $\alpha_{m_{i_{1}}}^{\left(i_{1}\right)}$ and as an $\alpha_{m_{i_{2}}}^{\left(i_{2}\right)}$ edge. 
Fig. 4 A realization of the $\alpha_{m_{1}}^{(1)}$ edge $(a, b)$ with $t=3, k_{1}=3$, $k_{2}=2, k_{3}=3, m_{1}=2, m_{2}=2$, $m_{3}=1$. Nodes to the left/right of each other are meant to represent the same element

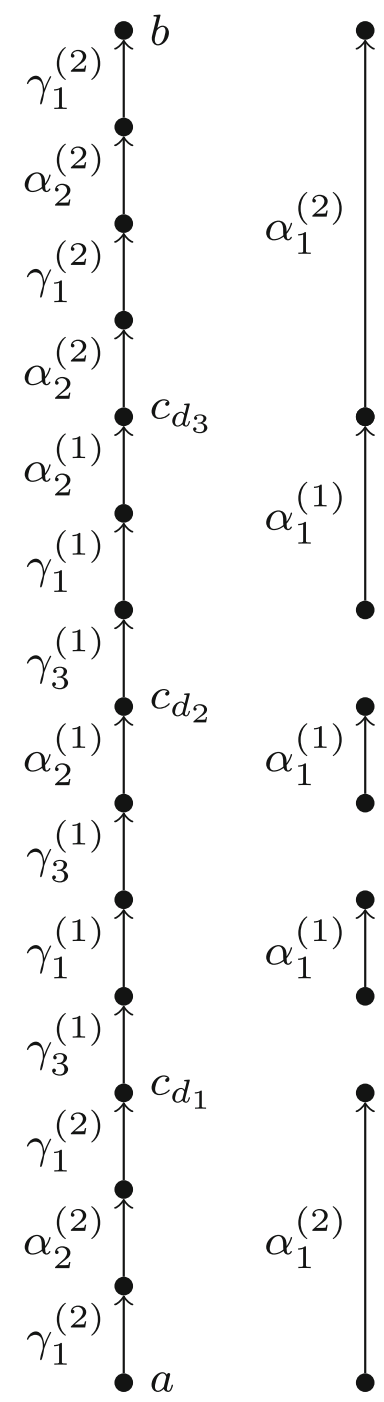

For an edge $\left(x_{1}, x_{2}\right) \in \alpha_{m_{i}}^{(i)}$, denote with $\mathcal{R}_{\left(x_{1}, x_{2}\right)}^{(i)}$ the set of the edges of the canonical realization of $\left(x_{1}, x_{2}\right)$ as an $\alpha_{m_{i}}^{(i)}$ edge, and set

$$
\mathcal{K}_{\left(x_{1}, x_{2}\right)}^{(i)}=\left\{\left(y_{1}, y_{2}\right) \in \mathcal{R}_{\left(x_{1}, x_{2}\right)}^{(i)}: y_{2} \neq x_{2},\left(y_{1}, y_{2}\right) \in \alpha_{m_{i}}^{(i)} \cup \alpha_{m_{i+1}}^{(i+1)}\right\}
$$

Define a rank of the edge $\left(x_{1}, x_{2}\right)$ : it will be zero if $\mathcal{K}_{\left(x_{1}, x_{2}\right)}^{(i)}=\emptyset$, otherwise, the rank is recursively defined as the maximal rank of the elements of $\mathcal{K}_{\left(x_{1}, x_{2}\right)}^{(i)}$ plus one. As $\mu$ satisfies DCC, all $\alpha_{m_{i}}^{(i)}$ edge has a (finite) rank. 
Take an $\alpha_{m_{1}}^{(1)}$ edge $\left(x_{1}, x_{2}\right)$ of rank 0 . In its canonical realization there is at most one edge that is also an $\alpha_{m_{1}}^{(1)}$ or an $\alpha_{m_{2}}^{(2)}$ edge (the last edge of the realization). This means that

$$
\begin{aligned}
\left(x_{1}, x_{2}\right) \in \eta_{0}^{(1)}:= & \left(\alpha _ { m _ { 1 } } ^ { ( 1 ) } \wedge \left(\left(\alpha_{1}^{(2)} \wedge\left(\gamma_{1}^{(2)} \vee\left(\alpha_{2}^{(2)} \wedge \cdots \vee\left(\alpha_{m_{2}-1}^{(2)} \wedge \gamma_{m_{2}-1}^{(2)}\right)\right)\right)\right)\right.\right. \\
& \left.\left.\vee\left(\alpha_{m_{1}+1}^{(1)} \wedge\left(\gamma_{m_{1}+1}^{(1)} \vee\left(\alpha_{m_{1}+2}^{(1)} \wedge \cdots \vee\left(\alpha_{m_{1}-1}^{(1)} \wedge\left(\gamma_{m_{1}-1}^{(1)} \vee \alpha_{m_{1}}^{(1)}\right)\right)\right)\right)\right)\right)\right) \\
& \vee\left(\alpha _ { m _ { 1 } } ^ { ( 1 ) } \wedge \left(\left(\alpha_{1}^{(2)} \wedge\left(\gamma_{1}^{(2)} \vee\left(\alpha_{2}^{(2)} \wedge \cdots \vee\left(\alpha_{m_{2}-1}^{(2)} \wedge\left(\gamma_{m_{2}-1}^{(2)} \vee \alpha_{m_{2}}^{(2)}\right)\right)\right)\right)\right)\right.\right. \\
& \left.\vee\left(\alpha_{m_{1}+1}^{(1)} \wedge\left(\gamma_{m_{1}+1}^{(1)} \vee\left(\alpha_{m_{1}+2}^{(1)} \wedge \cdots \vee\left(\alpha_{m_{1}-1}^{(1)} \wedge \gamma_{m_{1}-1}^{(1)}\right)\right)\right)\right)\right) .
\end{aligned}
$$

We likewise define the $L$-elements $\eta_{0}^{(i)}$ for all $1 \leq i \leq t$, with each containing all the $\alpha_{m_{i}}^{(i)}$ edges of rank 0 . Note that the long expression above is not to be read as one would initially think: it is not a join of four subexpression, but two, and each of those two is a meet of $\alpha_{m_{1}}^{(1)}$ and an other (lengthy) expression.

We recursively define $L$-elements $\eta_{n}^{(i)} \leq \alpha_{m_{i}}^{(i)}$ for all $1 \leq i \leq t$ and all nonnegative integer $n$ : for $n=0$ they are already defined, and for $n>0$ we set

$$
\begin{aligned}
\eta_{n}^{(i)}:= & \left(\alpha _ { m _ { i } } ^ { ( i ) } \wedge \left(\left(\alpha_{1}^{(i+1)} \wedge\left(\gamma_{1}^{(i+1)} \vee\left(\alpha_{2}^{(i+1)} \wedge \cdots \vee\left(\alpha_{m_{i+1}-1}^{(i+1)} \wedge\left(\gamma_{m_{i+1}-1}^{(i+1)} \vee \eta_{n-1}^{(i+1)}\right)\right)\right)\right)\right)\right.\right. \\
& \left.\vee\left(\alpha_{m_{i}+1}^{(i)} \wedge\left(\gamma_{m_{i}+1}^{(i)} \vee\left(\alpha_{m_{i}+2}^{(i)} \wedge \cdots \vee\left(\alpha_{m_{i}-1}^{(i)} \wedge\left(\gamma_{m_{i}-1}^{(i)} \vee \alpha_{m_{i}}^{(i)}\right)\right)\right)\right)\right)\right) \\
& \vee\left(\alpha _ { m _ { i } } ^ { ( i ) } \wedge \left(\left(\alpha_{1}^{(i+1)} \wedge\left(\gamma_{1}^{(i+1)} \vee\left(\alpha_{2}^{(i+1)} \wedge \cdots \vee\left(\alpha_{m_{i+1}-1}^{(i+1)} \wedge\left(\gamma_{m_{i+1}-1}^{(i+1)} \vee \alpha_{m_{i+1}}^{(i+1)}\right)\right)\right)\right)\right)\right.\right. \\
& \left.\vee\left(\alpha_{m_{i}+1}^{(i)} \wedge\left(\gamma_{m_{i}+1}^{(i)} \vee\left(\alpha_{m_{i}+2}^{(i)} \wedge \cdots \vee\left(\alpha_{m_{i}-1}^{(i)} \wedge\left(\gamma_{m_{i}-1}^{(i)} \vee \eta_{n-1}^{(i)}\right)\right)\right)\right)\right)\right) .
\end{aligned}
$$

If $\left(x_{1}, x_{2}\right)$ is an $\alpha_{m_{i}}^{(i)}$ edge $\left(x_{1}, x_{2}\right)$ of rank $n$, then in its canonical realization all the $\alpha_{m_{i}}^{(i)}$ and $\alpha_{m_{i+1}}^{(i+1)}$ edges except perhaps one have rank at most $n-1$. From this fact we can easily prove by induction that $\left(x_{1}, x_{2}\right) \in \eta_{n}^{(i)}$.

As $\alpha_{m_{i}}^{(i)}$ is a completely join irreducible element of $\mathbf{L}$, and it is the union of all $\eta_{n}^{(i)}$ (as they form an increasing chain, and each $\alpha_{m_{i}}^{(i)}$ edge has finite rank), there is an $n_{i}$ so that $\alpha_{m_{i}}^{(i)}=\eta_{n_{i}}^{(i)}$. We can suppose that among all the $n_{i}, n_{1}$ is (one of) the smallest.

As $\eta_{n_{1}}^{(1)}$ is defined as the join of two elements of $L$ that are smaller or equal than $\alpha_{m_{1}}^{(1)}$, and $\alpha_{m_{1}}^{(1)}$ is join irreducible in $\mathbf{L}, \alpha_{m_{1}}^{(1)}$ is equal to either

$$
\begin{aligned}
& \alpha_{m_{1}}^{(1)} \wedge\left(\left(\alpha_{1}^{(2)} \wedge\left(\gamma_{1}^{(2)} \vee\left(\alpha_{2}^{(2)} \wedge \cdots \vee\left(\alpha_{m_{2}-1}^{(2)} \wedge\left(\gamma_{m_{2}-1}^{(2)} \vee \eta_{n_{1}-1}^{(2)}\right)\right)\right)\right)\right)\right. \\
&\left.\vee \quad\left(\alpha_{m_{1}+1}^{(1)} \wedge\left(\gamma_{m_{1}+1}^{(1)} \vee\left(\alpha_{m_{1}+2}^{(1)} \wedge \cdots \vee\left(\alpha_{m_{1}-1}^{(1)} \wedge\left(\gamma_{m_{1}-1}^{(1)} \vee \alpha_{m_{1}}^{(1)}\right)\right)\right)\right)\right)\right)
\end{aligned}
$$

or

$$
\begin{aligned}
& \alpha_{m_{1}}^{(1)} \wedge\left(\left(\alpha_{1}^{(2)} \wedge\left(\gamma_{1}^{(2)} \vee\left(\alpha_{2}^{(2)} \wedge \cdots \vee\left(\alpha_{m_{2}-1}^{(2)} \wedge\left(\gamma_{m_{2}-1}^{(2)} \vee \alpha_{m_{2}}^{(2)}\right)\right)\right)\right)\right)\right. \\
& \vee \quad\left(\alpha_{m_{1}+1}^{(1)} \wedge\left(\gamma_{m_{1}+1}^{(1)} \vee\left(\alpha_{m_{1}+2}^{(1)} \wedge \cdots \vee\left(\alpha_{m_{1}-1}^{(1)} \wedge\left(\gamma_{m_{1}-1}^{(1)} \vee \eta_{n_{1}-1}^{(1)}\right)\right)\right)\right)\right) .
\end{aligned}
$$

From both equalities follows an inequality of the type

$$
\alpha_{m_{1}}^{(1)} \leq\left(\alpha_{1}^{(2)} \wedge \delta_{1}\right) \vee\left(\alpha_{m_{1}+1}^{(1)} \wedge \delta_{2}\right) .
$$

Recall the definition of the $\alpha_{j}^{(i)}$ to see that a consequence of this is that $\alpha_{1}^{(2)} \leq \delta_{1}$ and $\alpha_{m_{1}+1}^{(1)} \leq \delta_{2}$.

From the $\alpha_{1}^{(2)} \leq \delta_{1}$ type inequality, we can deduce in the first case that

$$
\alpha_{m_{1}-1}^{(1)} \leq \gamma_{m_{1}-1}^{(1)} \vee \eta_{n_{1}-1}^{(1)}
$$


and in the second that

$$
\alpha_{m_{1}-1}^{(1)} \leq \gamma_{m_{1}-1}^{(1)} \vee \eta_{n_{1}-1}^{(1)} .
$$

Both are impossible: by the choice of $n_{1}, \eta_{n_{1}-1}^{(2)} \leq\left(\alpha_{m_{2}}^{(2)}\right)_{*}$ and $\eta_{n_{1}-1}^{(1)}<\left(\alpha_{m_{1}}^{(1)}\right)_{*}$, so either of these inequalities contradicts the fact that $\alpha_{j}^{(i)} \not \leq \gamma_{j}^{(i)} \vee\left(\alpha_{j+1}^{(i)}\right)_{*}$ is satisfied by all possible $i$ and $j$.

Problem 2 Is it true that a lattice $\mathbf{L}$ is representable with DCC posets if and only if $E_{\mathbf{K}}$ contains no cycle for any $\mathbf{K} \leq \mathbf{L}$ ? Is it true if, moreover, $\mathbf{L}$ is finite?

Here is an overview of the known properties of the class of representable lattices.

Theorem 3 For the class $\mathcal{R}$ of lattices representable with DCC posets:

- $\mathcal{R}$ is closed under taking sublattices and products,

- $\mathcal{R}$ does not contain the lattice $\mathbf{M}_{3}$,

- $\mathcal{R}$ contains all lower bounded lattices,

- even the finite part of $\mathcal{R}$ is not contained in $S D_{\vee}$,

- it is algorithmically decidable if a finite lattice $\mathbf{L}$ is in $\mathcal{R}$,

- if a lattice $\mathbf{L}$ is in $\mathcal{R}$, then $E_{\mathbf{L}}$ contains no cycle.

Proof The last two statements have been proved. $\mathbf{M}_{3}$ fails the condition of the last statement, because the three middle elements form a $D$-cycle, and there is an E-loop on that cycle. As it was mentioned in the introduction, all lower bounded lattices are representable with posets satisfying both DCC and ACC by Theorem 4.6 of [6].

For the statement about join semidistributivity, consider the lattice $\mathbf{D}_{2}$ (Fig. 1). It is not join semidistributive, but we can show that it is in $\mathcal{R}$. This can be done either by following through on the construction described in the beginning of Section 3, or by directly applying Theorem 1: it is easy to check that $\mathcal{C}_{\mathbf{D}_{2}}=\{(c, a, d),(c, d, a),(d, b, c),(d, c, b)\}$, and we can define $s$ so it maps the first two elements of $\mathcal{C}_{\mathbf{D}_{2}}$ to $c$ and the second two to $d$. Now

$$
T=\{(c, a),(d, b)\}
$$

and

$$
U=\{(a, a),(b, b),(c, c),(c, d),(d, c),(d, d)\},
$$

so indeed $U \circ T$ does not contain a cycle.

The only item left is that $\mathcal{R}$ is closed to direct products. If $\mathbf{L}_{i} \in \mathcal{R}$ for all $i \in I$ such that $\mathbf{L}_{i}$ is represented by DCC posets on $X_{i}$ (which can be assumed to be disjoint), $\prod_{i \in I} \mathbf{L}_{i}$ can be represented on $\bigcup_{i \in I} X_{i}$ : the element $\left(l_{i}\right)_{i \in I}$ will be represented by the (disjoint) union of the posets representing the individual $l_{i}$.

Problem 3 Is it true that $\mathcal{R}$ contains $S D_{\vee}$ ? Is there a nontrivial lattice quasi-identity satisfied by all members of $\mathcal{R}$ ?

Acknowledgments The author's research was partially supported by the Hungarian National Foundation for Scientific Research (OTKA) grant no. K115518. and the National Science Centre of Poland grant no. 2014/2013/B/ST6/01812

Funding Information Open access funding provided by University of Szeged (SZTE). 
Open Access This article is distributed under the terms of the Creative Commons Attribution 4.0 International License (http://creativecommons.org/licenses/by/4.0/), which permits unrestricted use, distribution, and reproduction in any medium, provided you give appropriate credit to the original author(s) and the source, provide a link to the Creative Commons license, and indicate if changes were made.

\section{References}

1. Schein, B.M.: A representation theorem for lattices. Algebra Univers. 2, 177-178 (1972)

2. Adaricheva, K.V., Gorbunov, V.A., Tumanov, V.I.: Join-semidistributive lattices and convex geometries. Adv. Math. 173(1), 1-49 (2003)

3. Caspard, N.: The lattice of permutations is bounded. Int. J. Algebra and Comput. 10(4), 481-490 (2000)

4. Freese, R., Ježek, J., Nation, J.B.: Free Latices, Mathematical Surveys and Monographs, vol. 42, Amer. Math. Soc., Providence, RI (1995)

5. Pudlák, P., Tůma, J.: Every finite lattice can be embedded in a finite partition lattice. Algebra Univers. 10, 74-95 (1980)

6. Semenova, M.V.: Lattices that are embeddable in suborder lattices. Algebra and Logic 44(4), 270-285 (2005)

7. Sivák, B.: Representations of finite lattices by orders on finite sets. Math. Slovaca 28(2), 203-215 (1978)

8. Whitman, P.M.: Lattices, equivalence relations, and subgroups. Bull. Amer. Math. Soc. 52, 507-522 (1946)

Publisher's Note Springer Nature remains neutral with regard to jurisdictional claims in published maps and institutional affiliations. 